\title{
Heart Rate Prediction Based on Physical Activity using Feedforward Neural Network
}

\author{
Ming Yuchi \\ School of Life Science and Technology \\ Huazhong University of Science and Technology \\ Wuhan, Hubei, China \\ m.yuchi@gmail.com \\ Jun Jo \\ School of Information and Communication Technology \\ Griffith University \\ Queensland, Australia \\ j.jo@griffith.edu.au
}

\begin{abstract}
The technique of combining heart rate $(H R)$ and physical activity $(P A)$ has been adopted in a number of research areas, such as energy expenditure measurement, autonomic nervous system assessment, sports research, etc. However, there have been few studies on the direct relationship between $H R$ and PA. This paper proposes a HR prediction model based on the relationship between $H R$ and PA. The predictor has the potential to be used in various areas, such as: cardiopathy research and diagnosis, heart attack warning indicator, sports capability measure and mental activity evaluation. The method has the following steps: first, the recorded $H R$ and $P A$ signals are preprocessed as two synchronized time sequences: $H R(n)$ and $P A(n)$. The inputs of the predictor are $H R(n)$ and $P A(n)$ in the current time step, and the output is the predicted sequence $H R(n+1)$ in the next time step. The Feedforward Neural Network (FFNN) was chosen as the mathematical model of the predictor. Experiments was conducted based on the real-life signals from a healthy male. A set of 90 minute signals were collected. One half of the signal set was used to train the FFNN and the other half to validate the training. The mean absolute error of the predicted heart rate was restricted inside 5. The result shows the potential of the proposed method.
\end{abstract}

\section{Introduction}

The heart rate (HR) is generally measured as a series of time intervals (the so-called RR intervals) between the heart cycles that are obtained from the electrocardiogram (ECG) [1]. Analysis on HR has become a popular noninvasive tool for the studies on cardiopathy and exercise physiology.

One limitation associated with HR monitoring technique is that it is difficult to identify whether the HR increase is due to physical activity (PA) or mental activity [2, 3], especially when the increase in heart rate is modest. One possible solution to this problem is to incorporate the PA signals into the investigation scope. Research projects and applications that combine HR and PA signals include: energy expenditure measurement [4] - [6], autonomic nervous system assessment [7] - [11], sports research [12] - [14].

Most of the above works utilize the HR and PA as two parallel inputs, and the output of the system is energy expenditure, oxygen consumption or nervous system routine. There have been only a few studies looking into the direct relationship between HR and PA. Pawar el al. [15] presented one body movement activity detection system which is based on ECG signal, but not HR. Meijer el al. [3] built a linear-type relationship between the HR and the body movement. However, the experiments were implemented within specific conditions and the body movement was recorded as the counted number of activities, which could not appropriately reflect the actual PA.

The main purpose of this paper is to build a HR prediction model, which is based on real-life HR and PA (3-D acceleration) signals. This model can be further developed 
to a HR abnormality detection system. For the experiment, the subject was equipped with the portable HR and PA monitor, then proceeded to perform normal daily activities without any special routine or restriction. The recorded HR and PA signals were preprocessed to produce two synchronized time sequences: $H R(n)$ and $P A(n)$. The inputs of the predictor are $H R(n)$ and $P A(n)$ in the current time step, and the output is the predicted sequence $H R(n+1)$ in the next time step.

Considering that all of the signals are non-constrained and real-time data, the predictor has the potential to be used in various areas, such as: cardiopathy research and diagnosis, heart attack warning indicator, sports capability measure and mental activity evaluation. One of the possible practical application is to integrate the predictor with the portable HR device to monitor asymptomatic HR sudden change, which is common for early-stage heart disease patient. The predictor compares the real HR and predicted one every time step, if the difference exceeds one predefined tolerance value, the device can warn the wearer or mark this part of ECG signal for diagnose reference.

The relationship between HR and PA is affected by many factors, such as age, sex, mental stress, ambient temperature hydration, etc. It is difficult to identify the direct rule behind the relationship. For this reason, we adopted feedforward neural network (FFNN) [16] - [18] as the mathematical model for the predictor, based on its intrinsic nonlinearity and computational simplicity.

This paper is organized as follows: firstly, the proposed method is presented in Section 2, which includes the introduction to the entire system, the signal recorder, the signal preprocessing and the FFNN. In Section 3, the predictor is tested with the signals obtained from one 33 year old healthy male. Finally, concluding remarks and discussions follow in Section 4.

\section{The Research Method}

\subsection{HR Prediction Model}

To investigate the relationship between the HR and PA, we need simultaneously-recorded HR and PA signals. One portable HR and PA monitor from Alive Technologies was used here. The monitor measures and records the wearer's ECG and PA (3-D acceleration) signals and determines the HR from the ECG in real-time. The left part of Fig. 1 shows the subject (user) wearing the monitor. The specification of the monitor will be described in Section 2.2.

The middle part of Fig. 1 is the preprocessor which converts the acquired HR $(h r(m))$ and acceleration signals $\left(a c_{x}(l), a c_{y}(l), a c_{z}(l)\right)$ into usable format. The outputs of the preprocessor include two synchronized sequences $H R(n)$ and $P A(n)$, which are forwarded to the FFNN as
Table 1. Data Specification of Alive Heart Monitor

\begin{tabular}{|c|c|c|}
\hline Signal & ECG & Accelerometer \\
\hline Channels/Axis & Single Channel & 3 Axes \\
Resolution & 8 bits & 8 bits \\
Sampling Rate & $300 \mathrm{samples} / \mathrm{sec}$ & $75 \mathrm{samples} / \mathrm{sec}$ \\
Dynamic Range & $-2.66 \mathrm{mV} \sim 2.66 \mathrm{mV}$ & $-2.7 \mathrm{~g} \sim 2.7 \mathrm{~g}$ \\
Bandwidth & $0.5 \mathrm{~Hz} \sim 90 \mathrm{~Hz}$ & $0 \mathrm{~Hz} \sim 20 \mathrm{~Hz}$ \\
\hline
\end{tabular}

inputs. The output of the neural network is $\widetilde{H R}(n+1)$, which is the predicted HR on next time step.

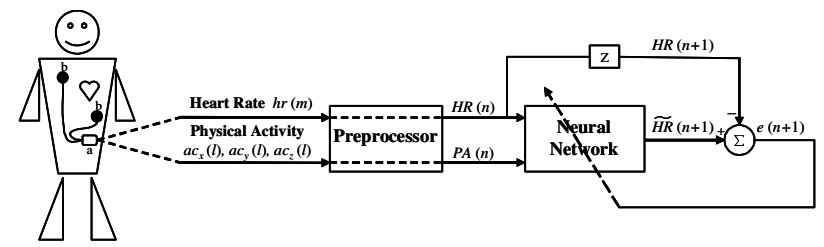

Figure 1. The block diagram of the whole system. a: a monitor which senses and records heart rates and physical activities; b: Electrodes.

\subsection{Heart Rate and Physical Activity Recorder}

Many studies on HR are based on the experimental data gathered in specific conditions and/or environments, whereas, this research was conducted with the data collected from normal daily activities, without requiring any pre-planned routine. Consequently, we need one portable device, which can monitor and record the HR and PA signals simultaneously for a period of time with relatively high accuracy.

According to the device requirements, one commercial product Alive Heart Monitor (AHM) is chosen for our experiments. Owing to its small size and light weight, the AHM can be worn comfortably during normal daily activities. The monitor gathers the single-channel ECG signal from a pair of electrodes attached at certain positions of the subjects's skin and 3-D physical activity (acceleration) signals from one build-in 3 axis accelerometer. The collected data can be saved in an internal SD memory card or transmitted to PC, smartphone or PDA using Bluetooth in real time. The AHM uses a rechargeable Lithium-ion battery which provides about four days of data saving or three days of wireless-transmission, continuously. The data specification of the AHM is shown in Table 1. 
Table 2. $H R(n)$ and $P A(n)$ Values of Fig. 3

\begin{tabular}{|c|c|c|}
\hline$n$ & $H R(n)(\mathrm{bpm})$ & $P A(n)(\mathrm{g})$ \\
\hline 1 & 122.5 & 0.62682 \\
2 & 121.5 & 0.66212 \\
3 & 122.75 & 0.67144 \\
\hline
\end{tabular}

The HR reading is generated on RR intervals of collected ECG signals. The sampling rate of HR is 1 samples/sec. Each HR is worked out as the average length of nine sequential RR intervals to reduce the influences of false or missing beat detections and ectopic beats.

Two examples of recorded signals are shown in Fig. 2, which includes the data of ECG, HR, acceleration of $x, y$ and $\mathrm{z}$ axes. Since the orientation of the axes may change along with the subject's movement, a certain amount of offset is added to each acceleration signal according to the orientation of the axis, which may help identify the body angles or the physical status of the subject.

While keeping a crouching posture in Fig. 2(a), the subject performs a jumping action between $1.5 s$ and $3 s$ in Fig. 2(b). It can be found that the jumping movement created some noises to the ECG signals, which may influence the accuracy of HR calculation.

\subsection{Signal Preprocessing}

The sampling rates of HR and acceleration are set differently in the AHM (1 samples/sec and 75 samples/sec, respectively) even though the inputs of the neural network are required to be sequences with same sampling rate. Here, we convert $h r(m)$ and $a c_{x}(l), a c_{y}(l), a c_{z}(l)$ into two synchronized sequences $H R(n)$ and $P A(n)$ through a processing $\operatorname{period} \tau$.

Assume the whole recording period is $T$, the recorded data on each signal channel are evenly divided into $N$ segments, each segment has the length of $\tau$,

$$
N=\operatorname{floor}(T / \tau),
$$

where floor $(x)$ rounds $x$ to the nearest integers towards minus infinity. The left part of Fig. 3 shows an example with $T=12 s$ and $\tau=4 s$. The recorded data are divided into $N=12 / 4=3$ segments on each channel. Each HR segment has $N_{h r}$ samples,

$$
N_{h r}=\text { samplingRate }_{h r} \times \tau ;
$$

and each acceleration segment has $N_{a c}$ samples,

$$
N_{a c}=\text { sampling Rate }_{a c} \times \tau .
$$

When $\tau=4 s$, HR segment has 1 samples/s $\times 4 s=4$ samples, and each acceleration segment has 75 samples/s $\times 4 s=300$ samples.

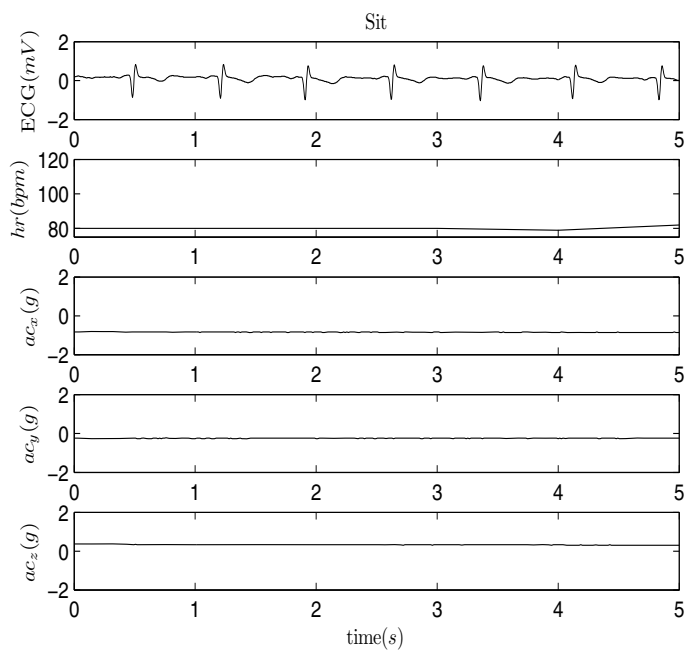

(a)

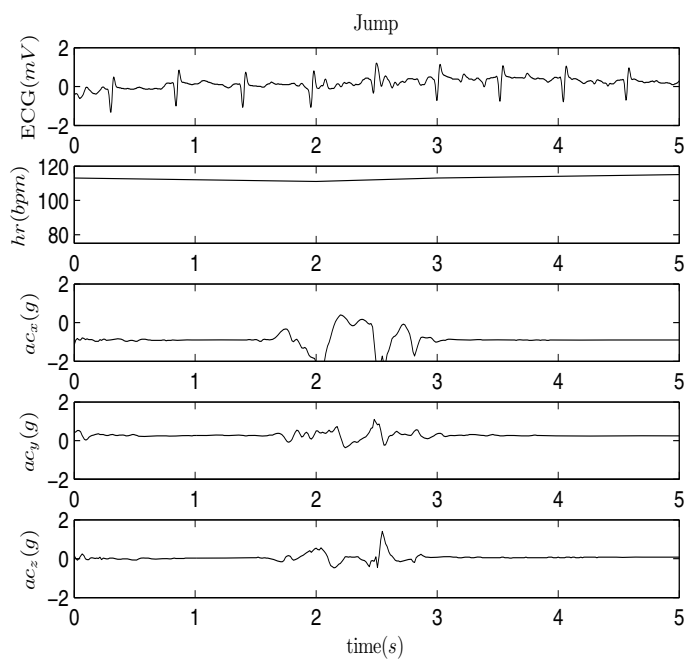

(b)

Figure 2. Examples of recorded AHM data: ECG, HR, Acceleration in x-axis, y-axis, zaxis. (a) Subject sat for five seconds; (b) Subject performed one jump action in five seconds. 


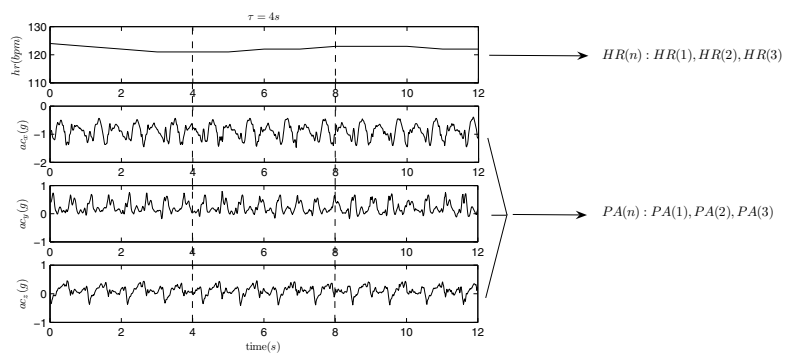

Figure 3. An example of Data preprocessing: $T=12 s$ and $\tau=4 s .3$ segments are formed on each channel. Then, these segments are converted into two synchronized sequences, $H R(n)$ and $P A(n)$.

Then, the $n$th $(n=1, \ldots, N) h r$ segment is converted into $H R(n)$, and the $n$th $a c_{x}, a c_{y}, a c_{z}$ segments are converted into $P A(n)$, through the following functions,

$$
H R(n)=\frac{\sum_{m=(n-1) * N_{h r}+1}^{n * N_{h r}} h r(m)}{N_{h r}},
$$

$$
\begin{aligned}
& P A(n)= \\
& \quad n * N_{a c}-1 \\
& l=(n-1) * N_{a c}+1
\end{aligned}
$$

$H R(n)$ is the average heart rate of $n$th segment. $P A(n)$ is also worked out as an average value. However, instead of the acceleration signals being directly used, the absolute difference value of adjacent acceleration signals is adopted to calculate $P A(n)$. This reflects the PA change between adjacent time steps, and eliminates the influences of the offset added on the acceleration signals. The right part of Fig. 3 shows the two sequences $H R(n)$ and $P A(n)$, and Table 2 lists the corresponding values.

It should be noted that the function of $\tau$ is not only to synchronize the inputs to neural network, but also to help stabilize the prediction accuracy through averaging the noises. This works well, especially when some signals have high noises, e.g., the jumping action in Fig. 2(b).

\subsection{Feed Forward Neural Network}

In this work, there exists two factors which increase the difficulty of the prediction. The first factor is that the subject performs normal daily activities. The consequence is that the recorded HR is influenced by different aspects, such as, the subject's body condition, mood and surrounding environment. The second factor is that the data is collected from one portable monitor. The accuracy and precision of the device may be limited compared to the equipment in a hospital laboratory.

These factors adds uncertainties to the experiments. In fact, $H R(n)$ and $P A(n), H R(n)$ and $H R(n+1)$ show nonlinear relationships in the data set obtained from the AHM, especially when $\tau$ is a relatively small value. Therefore, a mathematical method aiming at nonlinear prediction is needed. FFNN appears to be a good candidate [19, 20]. With a certain structure, multi-layer FFNN can be used as a general function approximator [21] - [23].

A FFNN [16] - [18] is a biologically inspired classification algorithm. It consist of a (possibly large) number of simple neuron-like processing units, organized in layers. Every unit in a layer is connected with all the units in the previous layer. These connections are not all equal, each connection may have a different strength or weight. The weights on these connections encode the knowledge of a network. Often the units in a neural network are also called neurons(nodes).

Data enters at the inputs and passes through the network, layer by layer, until it arrives at the outputs. During normal operation, there is no feedback between layers. This is why they are called feedforward neural networks.

Without needing any mathematical knowledge between the input and output, the FFNN is trained based on comparisons of the output and the target, until the network matches the target (Fig. 1).

\section{Pilot Experiment}

\subsection{Experiment Specifications}

In this paper, the subject was chosen as a 33 years male with no record of heart disease. The recording time period was 90 minutes. During this continuous period, the subject wore an AHM and performed the following activities: sitting and reading on the sofa, walking to the bus station, running to catch a bus, sitting in the bus, walking to the office, and other normal actions in his office.

The recorded signals were evenly separated as two parts. The first 45 minute signals were adopted as the training set, which was used to train the FFNN. The remaining 45 minute signals were for the test set, which was used to validate the trained neural network. The preprocessing parameter $\tau$ can be set with different values based on the user's practical desire. Here, $\tau$ was set to be $30 s$ for experiment test. Various values of $\tau$ will be tested in the future work. Therefore, for both the training and test sets, $N=90$ in Fig. 4 and Fig. 5 elucidate the corresponding $H R(n)$ and $P A(n)$ of the training set and test test.

The Neural Network Toolbox of the Matlab 7 was chosen to generate and train the neural network. Two-layer FFNN was selected as the predictor for this experiment. The two 

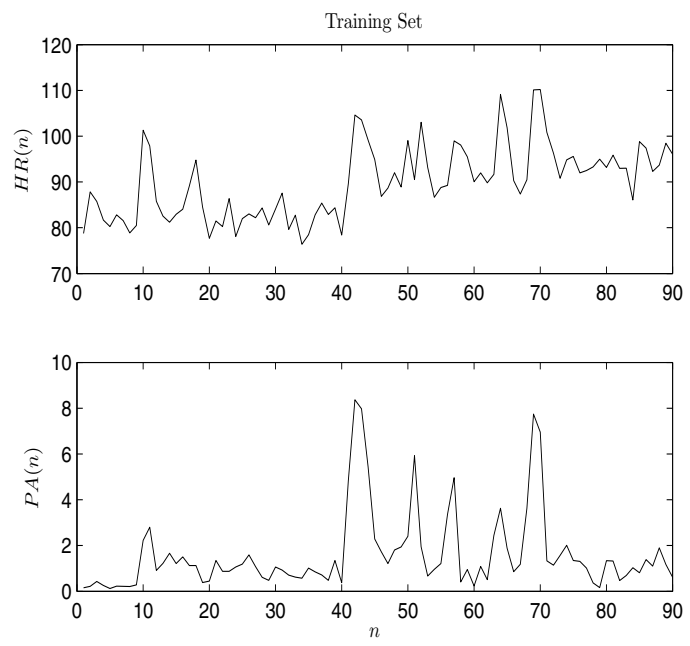

Figure 4. Training set for neural network training, $T=45 \mathrm{~min}, \tau=30 \mathrm{~s}, N=90$.

inputs of the FFNN were $H R(n)$ and $P A(n)$. The output layer (the last layer) had one neuron, $\overparen{H R}(n+1)$, the predicted HR of the next time step. The hidden layer (first layer) had 50 neurons. The number of hidden neurons is selected based on test-and-trial method. Fig. 6 shows the structure of the FFNN used in this paper.

Normally, the FFNN is trained with a backpropagation method, which includes many variations. Here, the Levenberg-Marquardt backpropagation method [24, 25] was adopted, based on its good performance and fast training speed for moderate-sized FFNN [26]. The network was trained for 200 generations on the training set.

\subsection{Experimental Results}

The performance of the neural network predictor on the training set and test set is shown in Fig. 7 and Fig. 8. To make a clear identification, the predicted $\widetilde{H R}(n+1)$ is denoted with a dashed line, while the original $H R(n+1)$ is represented by a unbroken line. The figures indicate that the $\widehat{H R}(n+1)$ follow the variance of $H R(n+1)$ on both the training set and test set after training.

The residual errors between the the $H R(n+1)$ and $\widetilde{H R}(n+1)$ are also shown in Fig. 7 and Fig. 8. The corresponding mean absolute error (MAE) and the variance of the error are listed in Table 3. Considering that the experiment was worked on real-life data and the prediction interval was only $30 s$, the MAE on both training and test sets is acceptable. However, the variance of the error is still relatively large. In Fig. 7 and Fig. 8, some residual errors are as big as 15 , although most of the residual errors are smaller
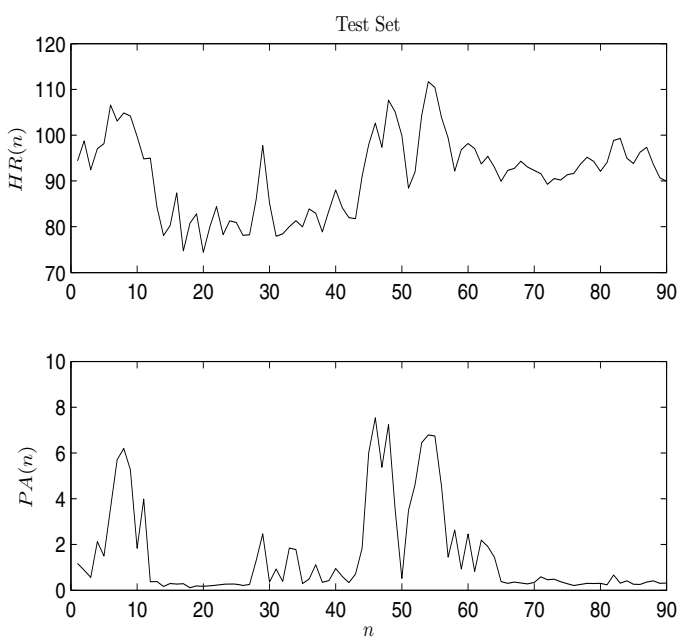

Figure 5. Test set for neural network validation, $T=45 \mathrm{~min}, \tau=30 \mathrm{~s}, N=90$.

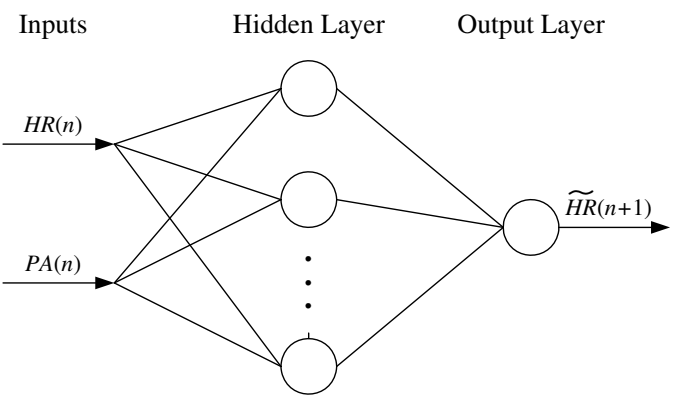

Figure 6. Two-layer FFNN structure.

than 5. Reducing the variance value to maintain the consistency of the prediction remains to be an objective of our future research.

\section{Conclusion and Discussion}

In this paper, a HR predictor based on PA was proposed. The predictor has the potential to be used in various areas, such as: cardiopathy research and diagnosis, heart attack warning indicator, sports capability measure and mental activity evaluation. FFNN was adopted as the mathematical model of the predictor. Experiments was conducted on 90 minutes of real life data were collected from one 33 year old healthy male who wore the heart monitor, AHM. The prediction was performed every 30 second. The result showed the potential of the predictor with the results close to the actual data. The mean absolute error could be restricted within a small range. The consistency of the prediction needs be 

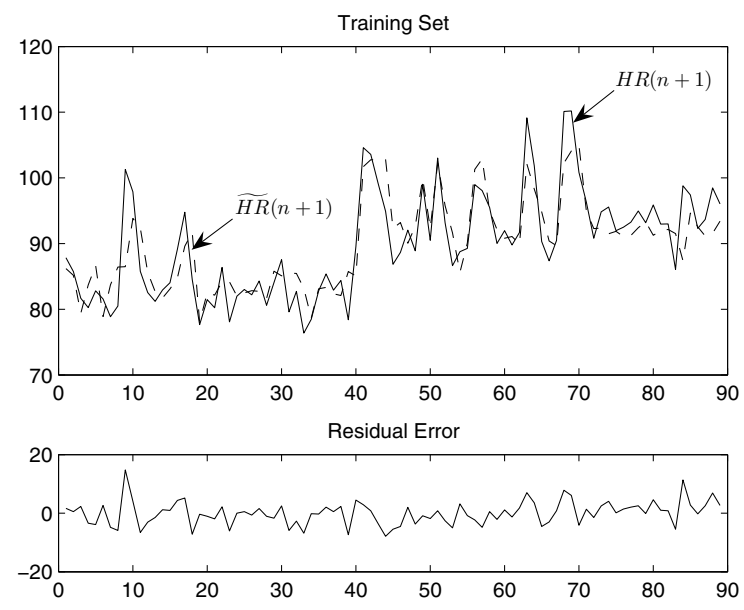

Figure 7. Feedforward neural network predictor performance on the training set: $H R(n+1)$ and $\widetilde{H R}(n+1)$, and the corresponding residual error.

\section{Table 3. MAE and Variance of the prediction} error

\begin{tabular}{|c|c|c|}
\hline Data Set & MAE & Variance \\
\hline Training Set & 3.12 & 16.62 \\
Test Set & 3.31 & 18.68 \\
\hline
\end{tabular}

improved and will be addressed in the future work.

To validate the effective of the proposed method and improve the neural network performance, further and deeper investigations are needed. Firstly, many and various subjects are needed. The current experiment was tested on one healthy male. Data from subjects of varying age, gender and health level should be tested. With assistance from the medical profession, the experiment could be implemented with hospital patients. Secondly, more tests on different system parameters and structures are needed. The possible varying factors include: prediction interval and total time length, data structure, neural network structure and training method.

\section{References}

[1] T. F. ESC/ASPE, "Heart rate variability: Standards of measurement, phisiological interpretation, and clinical use," Circulation, vol. 93, pp. 1043-1065, 1996.

[2] W. L. Haskell, M. C. Yee, A. Evans, and P. J. Irby, "Simultaneous measurement of heart rate and body mo-
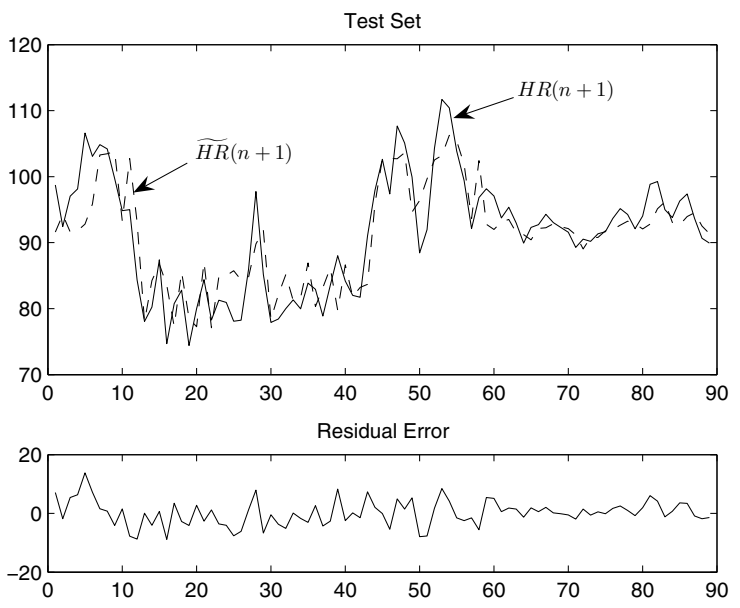

Figure 8. Feedforward neural network predictor performance on the test set: $H R(n+1)$ and $\widetilde{H R}(n+1)$, and the corresponding residual error.

tion to quantitate physical activity," Med. Sci. Sports Exerc., vol. 25, pp. 109-115, 1993.

[3] G. A. Meijer, K. R. Websterp, H. Koper, and F. ten Hoor, "Assessment of energy expenditure by recording heart rate and body acceleration," Medicine and Science in Sports and Exercise, vol. 21, pp. 343-347, 1989.

[4] K. Rennie, T. Rowsell, S. A. Jebb, D. Holburn, and N. J. Wareham, "A combined heart rate and movement sensor: proof of concept and preliminary testing study," European Journal of Clinical Nutrition, vol. 54, pp. 409-414, 2000.

[5] S. Brage, N. Brage, P. W. Franks, U. Ekelund, M.-Y. Wong, L. B. Andersen, K. Froberg, and N. J. Wareham, "Branched equation modeling of simultaneous accelerometry and heart rate monitoring improves estimate of directly measured physical activity energy expenditure," Journal of Appllied Physiology, vol. 96, pp. 343-351, 2004.

[6] J. K. Moon and N. F. Butte, "Combined heart rate and activity improve estimates of oxygen consumption and carbon dioxide production rates," Journal of Appliied Physiology, vol. 81, pp. 1754-1761, 1996.

[7] H. L. Chan, M. A. Lin, P. K. Chao, and C. H. Lin, "Correlates of the shift in heart rate variability with postures and walking by time frequency analysis," 
Computer Methods and Programs in Biomedicine, vol. 86, pp. 124-130, 2007.

[8] H. L. Chan, S. C. Fang, Y. L. Ko, M. A. Lin, H. H. Huang, and C. Lin, "Heart rate variability characterization in daily physical activity using wavelet analysis and multi-layer fuzzy activity clustering," IEEE Trans. Biomed. Eng., vol. 53, pp. 133-139, 2006.

[9] R. Perini and A. Veicsteinas, "Heart rate variability and autonomic activity at rest and during exercise in various physiologicalconditions," Eur. J. Appl. Physiol., vol. 90, pp. 317-325, 2003.

[10] R. Perini, S. Milesi, N. M. Fisher, and D. R. Pendergast, "A. veicsteinas, heart rate variability during dynamic exercise in elderly males and females," Eur. $J$. Appl. Physiol., vol. 82, pp. 8-15, 2000.

[11] L. Mourot, M. Bouhaddi, S. Perrey, J. D. Rouillon, and J. Regnard, "Quantitative poincaré plot analysis of heart rate variability: effect of endurance training," Eur. J. Appl. Physiol., vol. 91, pp. 79-87, 2004.

[12] L. H. Epstein, R. A. Paluch, L. E. Kalakanis, G. S. Goldfield, F. J. Cerny, and J. N. Roemmich, "How much activity do youth get? a quantitative review of heart-rate measured activity," Pediatrics, vol. 108, no. 3, pp. 1-10, 2001.

[13] W. Wang, J. R. Wei, D. C. Zhang, S. Z. Xiao, and F. C. Wang, "A study on 6-minute walk test incorporating cardiac contractility and heart rate change measurements," Chinese Medical Equipment Journal, vol. 24, no. 6, pp. 16-18, 2003.

[14] Y. S. Qin, M. Zhang, Z. G. Hao, and L. X. Song, "The use of the heart rate in training," Chinese Journal of Clinical Rehabilitation, vol. 7, no. 6, pp. 952-953, 2003.

[15] T. Pawar, S. Chaudhuri, and S. P. Duttagupta, "Body movement activity recognition for ambulatory cardiac monitoring," IEEE Trans. Biomed. Eng., vol. 54, no. 5, pp. 874-882, 2007.

[16] M. T. Hagan, H. B. Demuth, and M. H. Beale, Neural Network Design. Boston: MA: PWS Publishing, 1996.

[17] A. J. Annema, Feed-Forward Neural Networks: Vector Decomposition Analysis, Modelling and Apalog Implementation. Boston: Springer, 1995.

[18] S. Haykin, Neural Networks - A Comprehensive Foundation. New York: Macmillan College Publication, 1994.
[19] W. Y. Huang and R. P. Lippmann, "Comparisons between neural net and conventional classifiers," Lincoln Laboratory, MIT, Tech. Rep., 1987.

[20] _ _ "Neural net and traditional classifiers," Lincoln Laboratory, MIT, Tech. Rep., 1987.

[21] K. Hornik, M. Stinchcombe, and H. White, "Multilayer feedforward networks are universal approximators," Neural Networks, vol. 2, pp. 359-366, 1989.

[22] E. K. Blum and L. K. Li, "Approximation theory and feedforward networks," Neural Networks, vol. 4, pp. 511-515, 1991 .

[23] F. Scarselli and A. C. Tsoi, "Universal approximation using feedforward neural networks: a survey of some existing methods, and some new results," Neural Networks, vol. 11, pp. 15-37, 1998.

[24] K. Levenberg, "A method for the solution of certain non-linear problems in least squares," Quart. Appl. Math., vol. 2, pp. 164-168, 1944.

[25] D. Marquardt, "An algorithm for least-squares estimation of nonlinear parameters," SIAM J. Appl. Math., vol. 11, pp. 431-441, 1963.

[26] M. T. Hagan and M. Menhaj, "Training feedforward networks with the marquardt algorithm," IEEE Transactions on Neural Networks, vol. 5, no. 6, pp. 989993, 1994. 